Questions vives

\section{Questions Vives}

Recherches en éducation

Vol. $6 n^{\circ} 12$ | 2009

Evaluer les enseignants et les formateurs. Comment ? Pourquoi ? Pour quoi?

\title{
Principes, modalités et enjeux de l'évaluation des activités des professeurs d'université au Québec
}

\section{Jean Bernatchez}

\section{(2) OpenEdition}

Journals

Édition électronique

URL : http://journals.openedition.org/questionsvives/370

DOI : $10.4000 /$ questionsvives. 370

ISBN : 978-2-8218-1084-6

ISSN : $1775-433 \mathrm{X}$

Éditeur

Université Aix-Marseille (AMU)

Édition imprimée

Date de publication : 6 juin 2009

Pagination : 13-27

ISBN : 978-2-912643-36-0

ISSN : 1635-4079

\section{Référence électronique}

Jean Bernatchez, «Principes, modalités et enjeux de l'évaluation des activités des professeurs d'université au Québec », Questions Vives [En ligne], Vol.6 n¹2 | 2009, mis en ligne le 01 janvier 2011, consulté le 19 avril 2019. URL : http://journals.openedition.org/questionsvives/370 ; DOI : 10.4000/ questionsvives.370

\section{(c) (i) (3)}

Questions Vives est mis à disposition selon les termes de la licence Creative Commons Attribution Pas d'Utilisation Commerciale - Pas de Modification 4.0 International. 


\section{Principes, modalités et enjeux de l'évaluation des activités des professeurs d'université au Québec}

\section{Jean Bernatchez ${ }^{1}$}

Résumé : L'article vise à dégager les principes (le pourquoi) et les modalités (le comment) de l'évaluation des activités des professeurs d'université au Québec. Nous retenons comme vecteurs les trois missions de l'université québécoise: l'enseignement, la recherche et le service à la collectivité. Notre réflexion est organisée autour de questions génériques inspirées par Abernot (1996). Mais auparavant, nous présentons le contexte ayant contribué à la formalisation de l'évaluation de ces activités. En conclusion, nous illustrons quelques enjeux. Ces évaluations contribuent à accroître la qualité, la pertinence et l'efficience des activités des professeurs d'université au Québec. Toutefois, ces concepts ne sont pas neutres et riment de plus en plus avec la volonté affirmée de mieux servir les intérêts du marché mondialisé.

Mots-Clés : évaluation, professeurs, universités, Québec, marché mondialisé.

Abstract: This article aims to present the principles (why) and methods (how) of the evaluation of the activities of university professors in Québec, using as points of reference the three missions of the university : teaching, research, and community service. Our paper is organized around generic questions inspired by Abernot (1996). First, we present the context having contributed to the formalization of the evaluation of the activities. In conclusion, we illustrate some of the possible impacts. These evaluations contribute to increased quality, relevance and efficiency of the professors' activities in Québec. However, these concepts are not neutral and increasingly concord with the expressed desire to better serve the interests of the world market.

Keywords: evaluation, professors, universities, Québec, world market.

${ }_{1}$ Ph.D, Professeur régulier en Sciences de l'éducation - Université du Québec à Rimouski - Groupe de recherche Apprentissage et socialisation (APPSO). 
Jean Bernatchez

Évaluation des activités des professeurs d'université au Québec

\section{Principes, modalités et enjeux de l'évaluation des activités des professeurs d'université au Québec}

\section{Introduction}

Au Québec en 2010, l'évaluation des enseignants des niveaux préscolaire, primaire et secondaire est un enjeu de la négociation de leur nouvelle convention de travail. Dans le champ transdisciplinaire de l'étude des politiques publiques, l'enjeu est un espace de sens où se cristallisent les alliances entre les acteurs, en fonction de leurs intérêts et de leurs valeurs (Lemieux, 2009). Aucun système uniforme d'évaluation des enseignants n'est en vigueur. L'État québécois croit que la solution réside dans une approche souple adaptée à chaque milieu (Dion-Viens, 2009). Le syndicat des enseignants n'adhère pas au modèle de l'évaluation des enseignants. II argumente que les programmes universitaires de formation des maîtres préparent à l'analyse réflexive, laquelle peut conduire à un accompagnement pédagogique fait dans une perspective de soutien à l'enseignant (Bernard, 2009).

Cet enjeu s'inscrit dans le contexte d'une professionnalisation accrue des enseignants. Le concept de professionnalisation fait référence à la revendication d'un statut social distinct dans la division du travail, mais exprime surtout l'idée de la construction des compétences nécessaires à l'exercice de la profession (MEQ, 2001). Inspiré par Paquay (1994) et Perrenoud (1999), le ministère de l'Éducation du Québec propose en 2001 un référentiel de compétences de la profession enseignante : " les référentiels de compétences apportent à la fois une note descriptive, dans la mesure où les compétences sont renseignées par des indicateurs, ainsi qu'une note prospective par l'effet d'attente suggéré » (Jorro, 2006, p.33). Douze compétences sont regroupées en quatre catégories : fondements, acte d'enseigner, contextes social et scolaire, identité professionnelle. Elles servent d'assises à l'accompagnement et à la supervision pédagogiques des enseignants.

À la jonction de l'école secondaire et de l'université loge le réseau collégial. Les collèges d'enseignement général et professionnel (CEGEP) offrent des programmes pré-universitaires de deux ans et des programmes techniques de trois ans conduisant à une qualification pour le marché de l'emploi. Un corpus d'activités fondamentales est commun aux deux filières. L'évaluation des activités des enseignants répond à une logique semblable à celle des ordres d'enseignement inférieurs, même si les CEGEP sont des établissements d'enseignement supérieur. Des politiques institutionnelles balisent les compétences attendues par rapport à l'enseignement et à la vie collégiale (Lavoie \& Ouellet, 2009).

Toutes les universités québécoises sont publiques. Celles dites traditionnelles, par exemple l'Université Laval et l'Université de Montréal, ont été fondées grâce à une charte privée. L'Université du Québec, un réseau de neuf établissements présents sur tout le territoire, a été créée par une loi. Le caractère public de toutes ses universités est une particularité québécoise à l'échelle de l'Amérique du Nord, tout comme l'existence du réseau des CEGEP. Les universités du Québec disposent néanmoins d'une grande autonomie, même si une tendance lourde implique de confier plus de pouvoir aux membres externes de leurs conseils d'administration. La ministre de l'Éducation étudie en 2010 la pertinence d'adopter le projet de loi 38 sur la gouvernance des universités. II a pour but de régir leur fonctionnement administratif en imposant que les membres externes soient majoritaires dans 
Evaluer les enseignants et les formateurs

Comment, pourquoi, pour quoi ?

leurs conseils d'administration. Le projet propose aussi des mécanismes de reddition de comptes inspirés de ceux de l'entreprise privée. La Fédération québécoise des professeures et professeurs d'université (FQPPU, 2009) dénonce avec force cette initiative: "Sous le prétexte d'une saine gestion, la loi vise un contrôle qui va bien au-delà de l'administration financière et s'étend à toutes les activités universitaires, qu'elles soient de nature pédagogique, scientifique ou sociale » (p.1).

Dans les universités québécoises, les pratiques d'évaluation des professeurs interpellent une panoplie d'acteurs et reposent sur de nombreuses modalités proposées au fil du temps, sans qu'elles ne soient associées à un cadre intégrateur. II est possible cependant de lier l'évaluation des activités des professeurs aux trois missions de l'université québécoise: l'enseignement, la recherche et le service à la collectivité. Notre article vise à dégager les principes (le pourquoi) et les modalités (le comment) de l'évaluation des activités des professeurs d'université en retenant comme vecteurs ces trois missions. Notre réflexion est organisée autour de questions génériques inspirées par Abernot (1996). Évaluer dans quel but, pourquoi et pour qui ? Qui sont les commanditaires et quelles sont leurs attentes ? Qui sont les évaluateurs et quelle est leur légitimité ? Quelles sont la nature et la forme de l'action? Quels sont les objets et les produits de l'évaluation? Avant de nous livrer à cet exercice, nous présentons le contexte de la formalisation de l'évaluation des activités des professeurs d'université au Québec.

Les matériaux utiles à la rédaction de cet article proviennent des résultats d'une recherche comparative de l'Alliance for International Higher Education Policy Studies portant sur les politiques publiques de l'enseignement supérieur en Amérique du Nord (Trottier \& Bernatchez, 2005). Au plan des méthodes, l'analyse documentaire, incluant celle d'un important corpus de littérature grise, 11 entrevues semi-dirigées avec les parties prenantes et l'observation participante ont été mises à contribution. Sur ce dernier plan, notre précédente pratique professionnelle nous a amené à participer à l'évaluation des programmes des établissements du réseau de l'Université du Québec (1989-2002), à rédiger sa première politique cadre d'évaluation périodique des programmes (1991), à définir les normes et à appliquer les modalités d'évaluation des enseignements à l'École nationale d'administration publique (ENAP) de l'Université du Québec (2002-2007).

Nous portons cependant un regard critique sur cette pratique professionnelle, nourri par la recherche et les lectures savantes. Considérant les principes et les modalités de l'évaluation des activités des professeurs d'université au Québec, la méthode prend souvent le pas sur l'esprit de la démarche (Jorro, 2009). À défaut d'un sens à donner à ces activités, un sens construit et reconnu par les professeurs, les valeurs du référentiel dominant s'imposent d'elles-mêmes (Muller, 2009) : performance, compétitivité, efficience, flexibilité et clientélisme caractérisent le modèle diffusé sous le nom d'économie du savoir.

\section{Contexte de la formalisation de l'évaluation des activités des professeurs}

L'évaluation des activités des professeurs d'université existe depuis aussi longtemps que l'université elle-même, considérant une définition générique du concept d'évaluation : porter un jugement sur la valeur de quelque chose ou de quelqu'un. La tradition universitaire québécoise est jeune. L'Université McGill, de langue anglaise, est fondée en 1829 et la première université francophone, l'Université Laval, l'est en 1852. Elles sont orientées vers la mission d'enseignement. Les qualités recherchées chez l'universitaire sont alors l'éloquence, 
Jean Bernatchez

Évaluation des activités des professeurs d'université au Québec

la compétence pédagogique et un fort sentiment d'appartenance à l'institution (Gingras, 1991). À l'aune de ces qualités, le professeur est jugé par ses étudiants et par ses supérieurs. L'université québécoise de l'époque s'inscrit dans le cadre du modèle anglais défini par Newman : l'université n'est pas un lieu de recherche, elle doit surtout servir à épanouir les esprits (Chadwick, 1989) selon les principes de la liberal education.

Le modèle allemand, inspiré par Humboldt, combine enseignement et recherche. II émerge en Europe à compter du début du XIXe siècle, aux États-Unis peu après, mais il tarde à s'imposer au Québec. L'Université McGill est pionnière à ce chapitre. À compter de 1870, certains de ses professeurs recrutés en Grande-Bretagne poursuivent leurs recherches à Montréal. En 1920, le Canada français rejoint la tendance avec la création de facultés de sciences à l'Université Laval et dans sa succursale devenue autonome en 1920, l'Université de Montréal. C'est à ce moment que le modèle du professeur-chercheur fait son apparition (Gingras, 2003). II implique que le professeur soit aussi un chercheur identifié à sa discipline et à ses pairs, ce qui conditionne le jugement porté sur lui et sur ses activités. Le concept de République de la science (Polanyi, 1962) traduit ce phénomène. Une main invisible guide la coordination des activités de chaque scientifique par ajustements mutuels. Le mérite scientifique est lié à trois critères: la plausibilité, la valeur scientifique et l'originalité des travaux. L'évaluation par les pairs, essentiellement sur la base de la qualité des travaux, conditionne la publication et l'octroi de ressources.

La Révolution tranquille des années 1960 consacre l'entrée du Québec dans la modernité. C'est à ce moment qu'un conflit entre les deux modèles apparaît. Fournier, Mathurin et Gingras (1988) utilisent le cas de l'évaluation du dossier de promotion d'un professeur en sciences de l'éducation d'une université montréalaise au cours des années 1970 pour illustrer le triomphe du second modèle sur le premier, sur la base d'un consensus qu'il est possible de déceler dans la convention collective de travail des professeurs. Cette convention délimite ce qu'il faut entendre par activités de recherche. Les écrits du professeur évalué sont jugés comme n'étant pas des publications de recherche. Le professeur essuie une série de refus à sa demande d'agrégation, qui confirmerait sa permanence d'emploi. En dernier recours, un arbitre tranche en se faisant épistémologue. Les écrits du professeur ne sont pas des œuvres de recherche et, en conséquence, il ne pourra exercer la profession : "l'arbitrage devient ici une sorte de rituel au cours duquel des professeurs acceptent de sacrifier l'un de leurs collègues pour démontrer à la communauté universitaire que leur faculté, où régnaient précédemment les pédagogues, est devenue une vraie faculté universitaire " (p.47).

À compter des années 1980, la volonté utilitariste s'inscrit de plus en plus étroitement dans l'activité de recherche universitaire au Québec, comme en témoignent les programmes de subvention des organismes pourvoyeurs qui délaissent la recherche libre pour financer la recherche orientée (Bernatchez, 2009). Le modèle allemand implique la combinaison de l'enseignement et de la recherche, mais surtout leur autonomie par rapport aux contraintes politiques, sociales et économiques. L'autonomie interne de l'université, c'est par exemple l'indépendance des sciences particulières par rapport à la philosophie, dont l'ambition de systématisation porte atteinte au particularisme des disciplines. L'autonomie externe, c'est la quête désintéressée du savoir et l'élaboration de la science pure, sans considération d'utilité (Renaut, 1995). L'université québécoise bascule dans le modèle américain, hybride des modèles anglais et allemand, mais les transgressant au chapitre de l'utilitarisme. Ce modèle 


\section{Evaluer les enseignants et les formateurs \\ Comment, pourquoi, pour quoi?}

est campé dans l'ouvrage de Kerr (1963) avec son concept de multiversity: "A university anywhere can aim no higher than to be as British as possible for the sake of the undergraduates, as German as possible for the sake of the graduates and the research personnel, as American as possible for the sake of the public at large - and as confused as possible for the sake of the preservation of the whole uneasy balance " (p.18). À cela s'ajoute une idée forte : l'université doit quitter sa tour d'ivoire et assumer ses responsabilités sociales (Bok, 1982).

La mission de service à la collectivité émerge au Québec dans ce contexte au cours des années 1980, portée par les universités et promue par le Conseil des universités, un organisme gouvernemental créé en 1968 dans le but de conseiller le ministre de l'Éducation sur ses orientations. En plus de ses missions traditionnelles (l'enseignement et la recherche), l'université devient un réservoir d'expertises au service de la collectivité. Les professeurs sont des savants, mais aussi des experts. Cette dimension, conforme à la finalité des sciences appliquées, devient prégnante dans les facultés de sciences et de génie, mais elle acquiert aussi une importance considérable dans les autres facultés, dont celles des humanités.

La décennie des années 1980 marque la création puis la généralisation des Bureaux de liaison entreprises-universités (BLEU) dans les universités québécoises (Bernatchez, 2005). Sur le modèle de l'Office of Technology Transfer (OTT) des universités américaines, le BLEU assure le transfert vers l'industrie des technologies issues de la recherche universitaire. La prise de brevets par les universités leur permet d'obtenir des redevances grâce à l'octroi de licences aux entreprises utilisatrices. L'autre cas de figure est la création d'entreprises dérivées détenues par les universités et par leurs partenaires. Trois sphères (universités, entreprises, État) se chevauchent dans cette nouvelle infrastructure du savoir, ce qui donne naissance à des organismes hybrides de développement de la connaissance, conformément au modèle théorique de la Triple Hélice (Etzkowitz \& Leydesdorff, 2000): sociétés de valorisation commerciale de la recherche, technopôles, incubateurs d'entreprises, etc.

L'utilitarisme est aussi en terrain fertile avec la recherche-action, cette dynamique par laquelle les universitaires étudient les problèmes vécus par des groupes afin de guider leurs actions (Legendre, 2005). Plusieurs concepts sont véhiculés pour traduire la nécessité du transfert université-milieu des connaissances issues de la recherche universitaire : utilisation de la recherche sur le social (Weiss, 1979), mobilisation du savoir (Conseil de recherches en sciences humaines du Canada) et innovation sociale (ministère de la Recherche, de la Science et de la Technologie du Québec). Le Rapport de la Commission Gulbenkian présidée par Wallerstein (1996) se présente aussi comme une contribution théorique à cette œuvre d'ouverture des sciences sociales.

L'évaluation par les pairs convient peu à cette nouvelle réalité, aussi ce sont des modes d'évaluation mixtes qui s'imposent graduellement. Aux critères de qualité et de validité scientifiques s'ajoutent ceux de pertinence sociale, économique, politique, voire ceux d'efficience. Les utilisateurs des résultats de la recherche sont invités à délibérer de la qualité, de la pertinence et de l'efficience des projets de recherche proposés. Cette tendance lourde observée dans l'univers de la recherche universitaire se remarque aussi du côté de l'enseignement. L'instrumentalisation se traduit alors par une professionnalisation des programmes de formation, accentuée surtout à compter de 1990.

Cela dit, après cette mise en contexte au plan des principes, nous présentons maintenant les modalités de l'évaluation des activités des professeurs d'université, en considérant 
Jean Bernatchez

Évaluation des activités des professeurs d'université au Québec

chacune des trois missions de l'université québécoise. Mais avant, précisons que trois facteurs contribuent à la formalisation de l'évaluation de ces activités.

(1) La professionnalisation de la formation et l'instrumentalisation de la recherche (Trottier \& Bernatchez, 2005) font qu'il est plus facile d'associer les réalisations universitaires à des produits en lien avec des livrables définis de façon opératoire en termes de valeur ajoutée ou d'innovation. Les modalités d'assurance qualité permettent ainsi de mesurer l'écart entre ce qui est attendu et ce qui est livré. Le modèle américain d'université, fondé sur l'utilitarisme, est dominant au Québec, sous l'impulsion de la mondialisation et par la pratique du benchmarking à la fois comme outil de planification et modalité d'assurance qualité.

(2) L'essor du corporatisme, induit par l'individualisme contemporain (Lipovetsky, 1983), contribue aussi à cette formalisation. Les associations étudiantes exercent un lobby efficace pour s'assurer que les activités qui leur sont vendues soient de qualité au plan scientifique et pertinentes au plan socioéconomique, dans la perspective d'une meilleure qualification pour le marché de l'emploi. Les syndicats de professeurs mesurent aussi l'ampleur des tâches de leurs membres et repèrent les contraintes de la profession dans un contexte où les attentes à leur endroit sont de plus en plus grandes (Bertrand, 2003). On retrouve maintenant dans les conventions collectives des professeurs d'universités et dans les procédures qui en découlent des attentes signifiées et des pondérations qui permettent d'évaluer et de moduler leurs activités d'enseignement, de recherche, de service à la collectivité et de gestion universitaire.

(3) Finalement, dans un contexte où toutes les universités québécoises sont publiques, les principes du Nouveau management public (NMP) sont appliqués suite à la modification de la Loi sur les établissements d'enseignement universitaire en 1995 et l'adoption en 2000 de la Loi sur l'administration publique. Le NMP est un mouvement managériel et politique qui vise à réformer la pratique de l'administration publique en remplaçant les principes d'organisation bureaucratique de type wébérien par ceux du management prévalant dans le secteur privé (Rouillard, 2003). Les préceptes du NMP (efficience, flexibilité, clientélisme) tendent ainsi à remplacer ceux de l'administration publique traditionnelle (transparence, probité, équité).

\section{L'évaluation de l'activité d'enseignement}

L'évaluation de l'activité d'enseignement vise à apprécier la qualité et la pertinence de la formation reçue par les étudiants, et à aider le professeur à bonifier sa pratique d'enseignement. Elle est la responsabilité de l'université, en collaboration avec les étudiants et les professeurs. Elle se vit dans le microcosme de la classe, mais aussi au sein du programme de formation. Des pratiques officieuses d'évaluation de l'enseignement ont cours également, mais la plupart ne sont ni légitimes, ni conduites selon les règles de l'art.

L'évaluation de l'enseignement débute dans la classe par l'adhésion des étudiants et du professeur, avant le début d'une activité, au contenu d'un plan de cours détaillé. Certaines universités proposent des modèles uniformes de plans de cours alors que d'autres permettent une certaine liberté en ce qui a trait à la forme. Ces plans de cours décrivent les objectifs du cours, les méthodes pédagogiques, les modes d'évaluation, la liste des lectures, les thèmes spécifiques à chaque rencontre, les grilles de correction des travaux, etc. La première rencontre est consacrée à la discussion du contenu du plan de cours. Le droit des étudiants de commenter ce plan, voire de le remettre en question, est reconnu. À la fin de cet exercice, le plan de cours devient le contrat qui lie mutuellement le professeur et les étudiants. En cas de litige, il a une portée juridique. Cette pratique, obligatoire dans toutes les 


\section{Evaluer les enseignants et les formateurs \\ Comment, pourquoi, pour quoi ?}

universités québécoises, contribue à ce que le cours soit plus structuré et à ce que les étudiants puissent mieux planifier leur travail.

Au terme de la session, les étudiants évaluent la formation reçue eu égard aux paramètres évoqués au plan de cours. Les questionnaires d'évaluation de l'enseignement sont administrés par l'université de façon électronique. Ils sont structurés autour d'items de type Likert, c'est-à-dire d'affirmations auxquelles l'étudiant indique son degré d'accord sur une échelle graduée entre tout à fait d'accord et pas du tout d'accord. Par exemple, le questionnaire utilisé à l'Université du Québec à Rimouski compte 24 affirmations regroupées en cinq sections: objectifs, contenu, pédagogie, travail demandé et jugement global. L'échelle Likert est graduée de 1 à 4 : plus la moyenne tend vers 1 (tout à fait d'accord avec les affirmations), plus l'évaluation est positive. L'anonymat des répondants et la confidentialité des données sont garantis : seuls le professeur et le responsable du programme y ont accès.

L'évaluation de l'enseignement peut être utile pour permettre à un professeur de bonifier son cours, sa prestation ou sa pédagogie. Par exemple, l'ex-premier ministre français Juppé a été professeur invité à l'École nationale d'administration publique (ENAP) de l'Université du Québec. La revue L'Express relate ainsi l'impact de cette évaluation sur son enseignement : " évalué en fin de session par ses étudiants, à l'instar des autres enseignants, Juppé s'est vu reprocher de n'avoir pas assez partagé ses expériences concrètes. À la seconde session, il s'est senti plus en confiance, a laissé tomber la langue de bois et a commencé à donner son point de vue » (Barbier \& Grégoire, 2006).

Cette pratique officielle de l'évaluation de l'enseignement coexiste avec des pratiques officieuses. Le degré de confidentialité des résultats est variable lorsque les associations étudiantes assument la responsabilité de l'opération. Dans certains cas, les résultats sont même affichés sur des babillards (réels ou électroniques), contribuant à hiérarchiser les professeurs selon le degré de satisfaction des étudiants à leur endroit. On retrouve aussi des interfaces électroniques permettant de coter les professeurs. La rigueur est rarement au rendez-vous. II s'agit souvent de sites Internet prétextes à rendre accessible un contenu qui attire les gens. D'ailleurs, Thomas \& Ryan (2006) ont enquêté sur cette question et concluent, pour le cas de RateMyProfessors.com, l'interface la plus populaire : " the site's real purpose is to drive traffic to the company's Swooks website, which sells textbooks ".

RateMyProfessors.com est une expérience initiée en 1999 par des étudiants de San José State University de Californie. Elle se répand ensuite au Canada et au Royaume-Uni. II y a sur ce site les fiches d'évaluation d'un million de professeurs de plus de 6000 établissements d'enseignement supérieur. Cependant, n'importe qui peut évaluer un professeur grâce à l'échelle Likert proposée et publier des commentaires, y compris les personnes qui ne sont pas concernées. Sutherland (2006) y voit là un abus de pouvoir. Le site est logé aux ÉtatsUnis, pays qui interprète de façon libérale le concept de liberté d'expression : «Edward Pettit, associate dean of Arts and Sciences at Augusta State University, said that because the school receives federal funding, these evaluations technically are covered by the Freedom of Information Act " (Lohr, 2003). Lagorio (2006) évoque les problèmes éthiques que cela engendre en citant le cas d'un professeur dont la vie est ruinée à cause de propos homophobes publiés sur le site. Les initiateurs de RateMyProfessors.com disposent des capitaux et des moyens techniques leur permettant d'étendre mondialement leur entreprise.

L'évaluation des enseignements se poursuit au sein du programme. Les universités québécoises sont tenues d'évaluer au moins une fois tous les dix ans tous leurs programmes 
Jean Bernatchez

Évaluation des activités des professeurs d'université au Québec

suivant un protocole qui reprend les éléments d'une politique cadre d'évaluation périodique à laquelle adhérent les universités. À l'aube des années 1990, l'État québécois confie ainsi aux universités la responsabilité d'évaluer elles-mêmes leurs programmes de formation. La Conférence des recteurs et des principaux des universités du Québec (CREPUQ) coordonne cette responsabilité collective. Elle regroupe toutes les universités québécoises et permet d'harmoniser les politiques et les pratiques. Elle est l'interlocuteur privilégié du gouvernement pour les questions se rapportant à la vie universitaire. Elle a adopté en 1991 la politique cadre d'évaluation périodique des programmes. Elle s'est dotée d'une Commission de vérification qui analyse la conformité des politiques des universités avec la politique cadre, et la conformité des pratiques des établissements avec leurs politiques institutionnelles. Toute évaluation doit intégrer trois perspectives : la perspective interne, c'est-à-dire l'autoévaluation des professeurs et des étudiants, la perspective externe, soit l'avis d'experts indépendants, et la perspective institutionnelle.

De nombreux critères servent à évaluer la qualité, la pertinence et l'efficience des programmes. Au plan de la qualité, les données relatives à l'effectif étudiant, aux taux de diplomation, à la nature et au volume des ressources professorales, humaines, matérielles et financières associées au programme sont passées au crible. Au plan de la pertinence, les critères se définissent en termes d'opportunité scientifique (par rapport aux progrès récents du champ d'étude), d'opportunité socioéconomique (par rapport aux besoins du marché de l'emploi), d'opportunité systémique (par rapport aux programmes comparables) et d'opportunité institutionnelle (par rapport aux priorités et ressources de l'institution). Au plan de l'efficience, la CREPUQ a mis en place une Commission sur les programmes en vue d'analyser la pertinence et la complémentarité de tous les programmes de même que les modalités de concertation dans une perspective de rationalisation. L'évaluation périodique d'un seul programme mobilise plusieurs personnes pendant 12 à 18 mois.

Également, tout nouveau projet de programme doit être soumis à une évaluation externe de sa qualité et de sa pertinence conduite par la Commission d'évaluation des projets de nouveaux programmes de la CREPUQ puis ensuite par le ministère de l'Éducation. La première instance vérifie la qualité et la pertinence du programme grâce à un mécanisme d'évaluation par des pairs et des représentants socioéconomiques. Le ministère évalue ensuite la pertinence de financer le programme. Ces évaluations se font sur la base d'un dossier substantiel où sont mises en évidence les qualités du corps professoral concerné, et d'une visite de l'établissement par les experts externes et les membres du Comité d'évaluation. À partir de l'instant où une université décide de proposer un nouveau programme jusqu'au moment où elle peut l'offrir, on compte un délai minimal de deux à trois ans et un investissement institutionnel de plusieurs dizaines de milliers de dollars.

\section{L'évaluation de l'activité de recherche}

L'évaluation de l'activité de recherche vise à s'assurer du respect par les professeurs des normes scientifiques et éthiques propres à leur champ de recherche. Elle est réalisée par des organismes externes, dans la perspective d'octroyer les ressources aux meilleurs chercheurs et de maximiser ainsi leur investissement, selon le principe de l'excellence auquel souscrivent tous les organismes subventionnaires du Québec et du Canada. La recherche scientifique est en effet une responsabilité partagée entre les paliers fédéral et provincial. L'enseignement supérieur et l'éducation sont de compétence provinciale mais le gouvernement fédéral 


\section{Evaluer les enseignants et les formateurs \\ Comment, pourquoi, pour quoi?}

intervient indirectement dans ces domaines parce qu'ils couvrent un large spectre de la vie en société. L'évaluation des activités de recherche souscrit aux principes de l'évaluation par les pairs en ce qui a trait aux intrants (octrois) et aux extrants (publications). De plus en plus cependant, des utilisateurs de résultats de la recherche sont impliqués dans les processus d'évaluation des demandes de subvention, préoccupés davantage par la dimension de la pertinence socioéconomique des propositions que par celle de leur qualité scientifique.

Les professeurs doivent réaliser des activités en lien avec les trois missions de l'université, activités qu'ils peuvent par contre moduler. Faire beaucoup de recherche peut justifier un dégrèvement de cours, c'est-à-dire n'offrir que trois cours plutôt que quatre, la charge annuelle normale. Un cours représente 45 heures d'enseignement. S'impliquer dans la gestion universitaire ou dans des activités de service à la collectivité peut aussi justifier un dégrèvement de cours. Il est possible à un professeur, au cours de sa carrière, de moduler sa tâche. À moins d'occuper un poste de gestionnaire universitaire à temps complet, l'activité de recherche est la seule qui doit absolument être inscrite à la tâche. En effet, il est fréquent qu'un chercheur performant obtienne plusieurs dégrèvements de cours, financés par ses octrois de recherche. Alors la tâche d'enseignement est confiée à des chargés de cours, soit des personnes avec qui l'université établit un contrat d'offre de cours. Les chargés de cours sont souvent des étudiants au doctorat ou des praticiens d'expérience. Ils sont syndiqués et un système de pointage permet aux plus anciens d'avoir préséance sur les autres.

Les professeurs doivent rendre compte de leur travail sur une base annuelle. Ce principe est universel mais les modalités varient d'une université à une autre. La tâche est généralement adoptée par les collègues professeurs d'un même département. Cela prend la forme d'un rapport d'activités de l'année précédente et du programme de travail de l'année suivante, déposés autour du 1er juin, moment charnière entre deux années universitaires. Une production jugée normale permet un avancement d'échelon automatique qui commande une augmentation de salaire. Par exemple, à l'Université du Québec à Rimouski en 2010, la convention collective des professeurs prévoit une échelle salariale en 35 échelons associés à un salaire annuel variant entre 45267 \$ (échelon 1) et 111963 \$ (échelon 35). Le salaire moyen d'un Québécois est légèrement inférieur à $40000 \$$. Une production exceptionnelle peut amener un avancement rapide dans l'échelle salariale des professeurs. Lorsqu'un professeur souhaite passer du statut de professeur adjoint à celui de professeur agrégé, ou du statut de professeur agrégé à celui de professeur titulaire, il doit soumettre son dossier à l'évaluation externe. Généralement, les professeurs d'université sont au moins évalués tous les cinq ans. Le contenu du dossier d'évaluation et de promotion est normé à partir de dispositions de la convention collective de travail. II consiste en un bilan quantitatif et qualitatif des activités de la période comprise entre le moment de sa plus récente évaluation et le moment présent, au plus cinq années.

Au Québec, chaque professeur est responsable d'obtenir le financement nécessaire à ses travaux de recherche. II doit pour ce faire soumettre aux organismes subventionnaires, de préférence avec des collègues, un projet de recherche souvent défini sur trois ans. Ce projet est évalué par les pairs sur la base de sa qualité scientifique et de la qualité du dossier de publications des chercheurs. Les taux de succès varient entre $25 \%$ et $50 \%$, selon les organismes et les programmes. Des critères de pertinence et d'efficience sont aussi utilisés par les organismes subventionnaires. Avant les années 1980, seule la qualité scientifique des projets était considérée. Les processus de recherche sont également évalués et encadrés 
Jean Bernatchez

Évaluation des activités des professeurs d'université au Québec

par les comités d'éthique, présents dans toutes les universités canadiennes, qui s'assurent du caractère éthique de chaque recherche impliquant des sujets humains.

Les conseils et autres organismes subventionnaires canadiens et québécois financent une bonne partie de la recherche universitaire. Ils proposent des programmes plus ou moins orientés vers des thématiques en lien avec les priorités nationales. Certains de leurs programmes s'adressent à des clientèles particulières, par exemple les nouveaux professeurs-chercheurs, dans une perspective de soutien à la relève. Leurs programmes sont de plus en plus orientés. C'est dire que la recherche libre et désintéressée d'un point de vue socioéconomique dispose de moins de créneaux de financement que la recherche orientée vers des priorités déclarées. Le modèle canadien des conseils subventionnaires est de type décentralisé et coordonné. II est décentralisé parce que les conseils peuvent fonctionner de façon autonome dans le cadre de leur mandat et de leur enveloppe budgétaire. II est coordonné parce que les conseils doivent tenir compte également des orientations contenues dans les politiques fédérales et dans les budgets annuels qui prévoient parfois des objectifs précis et des enveloppes budgétaires dédiées. Au cours des récentes années, toute injection d'argent neuf dans les activités des conseils est conditionnée par l'atteinte d'objectifs construits à partir de priorités politiques, le plus souvent orientées vers le renforcement de la compétitivité de l'économie nationale dans le contexte de l'économie du savoir.

Malgré la rigueur de toutes ces évaluations, le marché de la recherche universitaire, tel que le conçoit Bourdieu (1984), demeure inégalitaire. Ce constat est partagé par des auteurs de toutes tendances qui ont exploré l'espace scientifique. Cole et Cole (1973) par exemple ont proposé une théorie de l'avantage cumulatif qui postule que la reconnaissance attire la reconnaissance. Zuckerman et Merton (1973) se sont intéressés aux facteurs extra scientifiques qui engendrent les inégalités de toutes natures. Rossiter (1993) a observé que les femmes tendent à être marginalisées dans l'espace scientifique. Bref, l'espace de la recherche universitaire recèle son lot d'inégalités. L'évaluation des activités de recherche n'évacue pas ces inégalités, elle contribue même souvent à les mettre en évidence.

\section{L'évaluation de l'activité de service à la collectivité}

La mission universitaire de service à la collectivité s'impose au Québec au cours des années 1980. À cette manière se développent les services liés à la culture, ceux par l'intermédiaire des professions, la formation continue, les partenariats tributaires de la volonté des universités de s'affirmer comme des acteurs sociaux dynamiques et engagés. L'activité de consultation doit être comprise dans le sens mélioratif qui associe l'université à un réservoir d'expertises au service de la société et de l'innovation technoscientifique. II s'agit d'une contribution, sollicitée ou non, réalisée en vertu de l'expertise de ses professeurs. Une proportion toujours plus importante de la recherche universitaire est liée à la recherche commanditée plutôt qu'à la recherche subventionnée. Les professeurs tendent à devenir des consultants plutôt que des savants.

Généralement, ce sont les utilisateurs des services qui évaluent la contribution des professeurs à ce chapitre. L'évaluation de l'activité de service à la collectivité se fait de plusieurs manières, considérant les multiples livrables: produits, services, interventions ou programmes. Cependant, le client est toujours associé à l'évaluation. Au plan sociétal, Nowotny et ses collègues (2003) développent l'idée d'agora. II s'agirait d'un nouvel espace public où se mêlent la science et la société, le marché et la politique. Les auteurs postulent 
Evaluer les enseignants et les formateurs

Comment, pourquoi, pour quoi ?

que cet endroit est le lieu des controverses relatives à la science, où débattent les membres de la société civile et les autres acteurs. L'agora contribuerait à l'acceptation sociale ou au rejet des innovations produites par la recherche. Le mouvement d'individualisation aurait introduit une conscience plus forte des pouvoirs devant appartenir aux individus, en mesure maintenant de relever ce défi puisque plus instruits, considérant qu'ils sont le produit d'un système de formation éclairé. Une telle agora, si elle existait et si elle était régie par des règles démocratiques, rejoindrait les préoccupations de ceux qui aspirent à une gouvernance collective et participative de la science, quoique la thèse des auteurs repose beaucoup sur la notion d'individualité et de rationalité. On peut aussi questionner l'influence relative des individus et des regroupements citoyens dans cette agora par rapport à celle des entreprises disposant de ressources importantes et pratiquant le lobbying de façon systématique.

L'université québécoise doit aussi rendre compte de ses réalisations à l'État, représentant des utilisateurs des services de l'université publique. Qualité, pertinence et efficience sont considérées. Un rapport est soumis annuellement au gouvernement (son contenu est normé par la loi), lequel doit être défendu en Commission parlementaire par le chef d'établissement. La Commission parlementaire sur l'éducation est composée de députés de l'Assemblée nationale, parlement de l'État québécois. Le modèle des contrats de performance entre l'État et les universités, expérimenté entre 2000 et 2003, a été abandonné (Bernatchez, 2003). II posait les bases d'un financement conditionnel à la bonne performance de l'établissement.

\section{Conclusion}

Concluons maintenant en illustrant quelques enjeux qui se dégagent des modalités d'évaluation des activités des professeurs d'université au Québec.

(1) L'évaluation de l'enseignement par les étudiants, réalisée selon les règles de l'art par l'université, à partir de modalités convenues conjointement par les personnes concernées, notamment les étudiants et les professeurs, peut avoir une incidence positive sur la qualité de l'enseignement dispensé. II faut par contre que cette évaluation soit faite de bonne foi par les étudiants et que le professeur soit ouvert à la critique. Ce n'est pas toujours le cas. Cette forme d'évaluation a cours de façon systématique dans les universités du Québec depuis la seconde moitié des années 1980, dans une forme qui n'a guère évolué, ce qui amène la routine et favorise la banalisation. Plusieurs étudiants boudent l'opération parce qu'ils ont l'impression que leur avis n'est pas pris en considération par les personnes responsables, dans le contexte où un grand nombre de professeurs bénéficient de la sécurité d'emploi. Le passage du questionnaire papier au questionnaire électronique ne se fait pas fait sans heurts. Des études démontrent que les taux de réponse en ligne varient entre $29 \%$ et $55 \%$, alors que les taux de réponse papier se situent entre $61 \%$ et $77 \%$ (Dommeyer \& al., 2004). Or, il faut que plus de $50 \%$ des étudiants répondent au questionnaire pour que l'évaluation puisse être inscrite au dossier d'évaluation du professeur.

(2) II existe en Amérique du Nord un mouvement d'inflation des diplômes. Alors qu'auparavant un premier cycle universitaire était suffisant pour accéder à une profession, maintenant, c'est le grade de deuxième cycle qui est requis. Cela n'est pas étranger à la baisse des exigences dans les cours et à la hausse des résultats des étudiants, attribuables du moins en partie à l'évaluation de l'enseignement par les étudiants. II est admis qu'un professeur moins exigeant obtient de meilleurs résultats à l'évaluation, d'autant que certaines 
Jean Bernatchez

Évaluation des activités des professeurs d'université au Québec

affirmations du questionnaire prédisposent à cela. La notation littérale est en vigueur dans les universités québécoises. L'échelle varie entre At (excellent) et $E$ (échec). Des points sont attribués à chaque note (de 4,3 à 0 ) et une moyenne cumulative de ces points illustre la performance de l'étudiant. Le professeur est aussi porté à attribuer d'excellentes notes à ses meilleurs étudiants afin qu'ils soient favorisés lors de l'octroi de bourses au mérite.

(3) L'évaluation périodique et systématique des programmes des universités du Québec est en vigueur depuis le début des années 1990. Cette évaluation est bien intégrée aux pratiques, mais elle commande de s'associer un personnel qualifié et nombreux pour supporter les responsables de programmes dans cette tâche. Cette évaluation amène des coûts importants. Des systèmes informatiques standardisés de suivi et des tableaux de bord de gestion sont développés. Cela tend à favoriser l'utilisation d'indicateurs quantitatifs pour apprécier l'état de santé d'un programme. De plus, les exigences de la politique cadre de la CREPUQ font d'insister beaucoup sur les aspects socioéconomiques, de sorte que l'évaluation induit naturellement une professionnalisation des programmes universitaires. II n'est plus suffisant de démontrer que les étudiants du programme de philosophie, par exemple, reçoivent une formation de qualité. II faut aussi démontrer qu'ils se trouveront un emploi au terme de leurs études, adapté aux compétences développées.

(4) L'évaluation par les pairs demeure le meilleur moyen d'apprécier la qualité scientifique des réalisations de recherche des professeurs. Le mécanisme n'est pas neutre cependant. Lamont (2009) a mené une enquête sur le sujet et conclut, à l'instar de la plupart des auteurs à s'être penchés sur la question avant elle, que l'évaluation par les pairs n'est pas qu'une expérience cognitive. Les émotions et les relations interpersonnelles y jouent un rôle déterminant. Les modalités d'évaluation d'un professeur pour la promotion en carrière et l'octroi de ressources reposent sur un jugement conditionné par sa production, souvent mesurée par le nombre de ses publications. S'ensuivent une pression sur les chercheurs, un jeu d'influence pour favoriser les proches collègues. Mulkay (1969) observe aussi que les chercheurs font généralement preuve d'une rigidité qui laisse peu de place à l'originalité. Ils recherchent le consensus cognitif au moment d'évaluer les productions de leurs collègues. Mitroff (1974) observe que plusieurs chercheurs trouvent normal de juger les travaux de leurs collègues sur la base de critères fondés sur leur personnalité.

(5) L'idée d'élargir la composition des comités d'évaluation des activités des professeurs d'université en y associant des utilisateurs est pertinente, quoiqu'elle risque de desservir les professeurs qui ne proposent pas des activités immédiatement utiles, eu égard aux schèmes qui motivent l'action des utilisateurs. Or, la recherche fondamentale, la réflexion critique et les arts contribuent aussi de façon tangible au mieux-être des individus et des sociétés. Force est d'admettre cependant que la plupart des utilisateurs conviés à participer aux mécanismes d'évaluation sont des gens préoccupés surtout à servir l'intérêt national, largement défini en termes d'économie et de concurrence. Cette forme d'engagement est peu adaptée à la logique du partage du savoir qui devrait pourtant caractériser l'université du XXle siècle.

Cela dit, toutes ces évaluations contribuent à accrôtre la qualité, la pertinence et l'efficience des activités universitaires. Toutefois, ces concepts riment de plus en plus avec la volonté de mieux servir les intérêts du marché mondialisé, selon le modèle promu par l'OCDE (1996) et diffusé sous le nom d'économie du savoir. Au Québec, la formation universitaire est plus que jamais orientée vers la finalité du développement du capital humain dont a besoin l'économie nationale pour demeurer concurrentielle. Cette finalité conditionne sa qualité et sa 
Evaluer les enseignants et les formateurs

Comment, pourquoi, pour quoi ?

pertinence. Lorsque l'activité est réalisée avec un minimum de ressources, on constate aussi son efficience, selon les principes du Nouveau Management Public (NMP) dont s'inspire l'État québécois. La recherche universitaire, de plus en plus, est orientée vers les besoins des clients. La recherche contractuelle, appliquée et orientée, gagne en importance par rapport à la recherche subventionnée, libre et désintéressée.

II existe cependant dans les universités québécoises des îlots de résistance à ce mouvement. Fontan (2000) associe ces îlots à la critique radicale et aux utopies réalistes. L'UNESCO (2005) propose une de ces utopies réalistes, modèle alternatif à celui de l'OCDE, anthropocentrique et diffusé sous le nom de sociétés du savoir, sociétés au pluriel pour bien rendre compte de la diversité culturelle. Ces sociétés doivent reposer sur les droits de la personne, le respect de la dignité humaine, la solidarité entre les peuples et en leur sein. L'UNESCO convie les États à privilégier trois types d'initiatives, autant de piliers pour instaurer ces sociétés du savoir : valoriser les savoirs existants et les savoirs locaux pour lutter contre la fracture scientifique; instaurer une approche participative à l'accès à la connaissance ; prévoir l'intégration des politiques du savoir et clarifier leurs finalités pour faire en sorte qu'elles soient convergentes avec celles du bien commun, de l'équité et de la justice sociale, de la paix et du développement durable.

\section{Bibliographie}

Abernot, Y. (1996). Les méthodes d'évaluation scolaire. Paris: Dunod.

Barbier C. \& Grégoire, I. (2006). Juppé, l'ami québécois. L'Express, 11 mai.

Bernard, M. (2009). Des propos empreints de préjugés. Le Soleil, 13 septembre.

Bernatchez, J. (2009). Référentiels et dynamiques des politiques publiques de l'organisation de la recherche universitaire au Québec. Québec: Université Laval.

Bernatchez, J. (2006). Principes, enjeux et modalités de l'assurance qualité des activités universitaires au Québec. Communication au Séminaire international sur les réformes universitaires, El Jadida (Maroc), 24 mai.

Bernatchez, J. (2005). Regard et réflexions sur la constitution d'un réseau universitaire de transfert de technologie: les Bureaux de liaison entreprises-universités (BLEU) du Québec depuis 1986. Communication au Forum franco-québécois sur la valorisation de la recherche publique au Québec et en Île-de-France, Réseau français C.U.R.I.E. de valorisation de la recherche publique, Paris (France), 15 septembre.

Bernatchez, J. (2003). L'expérience québécoise de la conclusion de contrats de performance entre l'État et les universités. Sciences de la société, 58, 152-169.

Bertrand, D. (2003). Diversité, continuité et transformation du travail professoral dans les universités québécoises (1991 et 2003). Sainte-Foy: Conseil supérieur de l'éducation.

Bok, D. (1984). Beyond the Ivory Tower. Social Responsabilities of the Modern University. Harvard: Harvard University Press.

Bourdieu, P. (1984). Homo Academicus. Paris: Minuit.

Chadwick, O. (1989). Newman. Paris: Cerf.

Cole, S. \& Cole, J. (1973). Social stratification in science. Chicago: University of Chicago Press.

Dion-Viens, D. (2009). Évaluation des enseignants. La ministre inquiète. Le Soleil, 13 septembre. 
Dommeyer, C., Baum, P., Hanna, R. W. \& Chapman, K. S. (2004). Gathering faculty teaching evaluations by in-class and online surveys: their effects on response rates and evaluations. Assessment and Evaluation in Higher Education, 29(5), 611-623.

Fontan, J.-M. (2000). De l'intellectuel critique au professionnel de service. Radioscopie de l'universitaire engagé. Montréal: UQAM.

Fournier, M., Mathurin, C. \& Gingras. Y. (1988). L'évaluation par les pairs et la définition légitime de la recherche. Actes de la recherche en sciences sociales, 74(1), 47-54.

Gingras, Y. (2003). Enseignement, recherche, innovation. Les sciences en mutation. Capaux-diamants, 72, 50-53.

Gingras, Y. (1991). L'institutionnalisation de la recherche en milieu universitaire et ses effets. Sociologie et sociétés, 23(1), 41-54.

Fédération québécoise des professeures et professeurs d'université (FQPPU) (2009). La FQPPU s'oppose au projet de loi 38 et réclame la tenue d'États généraux sur l'université québécoise, Montréal: FQPPU.

Jorro, A. (2009). La reconnaissance en éducation. Évaluer, valoriser, légitimer. Ottawa: Presses de l'Université d'Ottawa.

Jorro, A. (2006). Devenir ami critique. Avec quelles compétences et quels gestes professionnels? Mesure et évaluation en éducation, 29(1), 31-44.

Lagorio, C. (2006). Hot for Teacher. Village Voice, January 11.

Lavoie, C. \& Ouellet, L. (2009). L'évaluation de tous les professeurs: les conditions d'une mise en œuvre prometteuse. Québec: Cégep de Ste-Foy.

Legendre, R. (2005). Dictionnaire actuel de l'éducation. Montréal: Guérin.

Lemieux, V. (2009). L'étude des politiques publiques. Les acteurs et leur pouvoir. Québec: Presses de l'Université Laval.

Lipovetsky, G. (1983). L'ère du vide. Essais sur l'individualisme contemporain. Paris:

Gallimard.

Lamont, M. (2009). How Professors Think. Inside the Curious World of Academic Judgment. Harvard: Harvard University Press.

Lohr, L. M. (2003). Site lets students grade professors. The Augusta Chronicle, January 15.

MEQ (Ministère de l'Éducation du Québec) (2001). La formation à l'enseignement. Les orientations. Les compétences professionnelles. Québec: MEQ.

Mitroff, I. (1974). The Subjective Side of Science: A Philosophical Inquiry into the Psychology of the Apollo Moon Scientifics. Amsterdam: Elsevier.

Mulkay, M. (1969). Some aspects of cultural growth in the natural science. Social Research, (36)1, $22-52$.

Muller, P. (2009). Les politiques publiques. Paris: Presses universitaires de France.

Nowotny, H., Scott, P. \& Gibbons, M. (2003). Repenser la science. Savoir et société à l'ère de l'incertitude. Paris: Belin.

OCDE (1996). L'économie fondée sur le savoir. Paris: OCDE

Paquay, L. (1994). Vers un référentiel de compétences professionnelles de l'enseignant? Recherche et formation, 15, 7-37.

Perrenoud, P. (1999). Dix nouvelles compétences pour enseigner. Paris: ESF.

Renaut, A. (1995). Les révolutions de l'université. Essai sur la modernisation de la culture. Paris: Calmann-Lévy. 
Evaluer les enseignants et les formateurs

Comment, pourquoi, pour quoi ?

Rossiter, M. W. (1993). The Matilda effect in science. Social studies of science. London: Sage.

Rouillard, C. (2003). Du cynisme au désabusement organisationnel. Le nouveau management public en tant que facteur de confusion. Choix, 9(6), 21-38.

Sutherland, J. (2006). Rank ratings. Education Guardian, April 12.

Thomas, O. \& Ryan, O. (2006). The telecom boom is back. CNNMoney.com, April 17.

Trottier, C., Bernatchez, J. (2005). Politiques publiques de l'enseignement supérieur au Québec: étude de cas. New York: AlHEPS.

UNESCO (2005). Vers les sociétés du savoir. Paris: UNESCO

Wallerstein, I. (1996). Ouvrir les sciences sociales. Paris: Descartes et Cie.

Weiss, C. H. (1979). The Many Meanings of Research. Public Administration Review, 39(5), 426-431.

Zuckerman, H. \& Merton, R. K. (1973). Institutionalized patterns of evaluation in science. In R. K. Merton (Ed.), The sociology of science. Chicago: University of Chicago Press. 